\title{
PSR J1913+1102: a pulsar in a highly asymmetric and relativistic double neutron star system
}

\author{
Robert D. Ferdman ${ }^{1}$ and the PALFA collaboration \\ ${ }^{1}$ Faculty of Science, University of East Anglia \\ Norwich Research Park, Norwich NR4 7TJ, United Kingdom \\ email: r.ferdman@uea.ac.uk
}

\begin{abstract}
PSR J1913+1102 is a double neutron star system (DNS) discovered in the Pulsar Arecibo L-band Feed Array survey. We have now very precisely measured the rate of advance of periastron for the system and the Einstein delay. From general relativity, this results in precise mass measurements: $1.65 \pm 0.05$ and $1.24 \pm 0.05 M_{\odot}$ for the pulsar and neutron-star companion, respectively. This makes PSR J1913+1102 both the most massive double neutron star system known, and the most asymmetric in mass among compact DNS binaries. This asymmetry will allow for stringent limits on the effects of dipolar gravitational-wave radiation, predicted by alternative theories of gravity, as well as insight into heavy-element production from the eventual merger of this system and others like it. Further observations will also tighten constraints on formation and evolution models; this is crucial for understanding the DNS population, for which there are relatively few mass measurements.
\end{abstract}

Keywords. (stars:) pulsars: general (stars:) pulsars: individual (PSR J1913+1102), stars: neutron, (stars:) binaries (including multiple): close, relativity, gravitation

\section{Introduction}

PSR J1913+1102 is a pulsar in a double neutron star (DNS) system, rotation with a 27.3-ms rotation period. Discovered in the PALFA survey by the Einstein@Home distributed computing project, it is a distant source; its dispersion measure of $339 \mathrm{~cm}^{-3} \mathrm{pc}$ implies an estimated $\sim 8 \mathrm{kpc}$ distance to the pulsar based on the modelled ionised electron content of the Galaxy (Cordes and Lazio 2003). It is also faint, having a $1400-\mathrm{MHz}$ flux density of $\sim 50 \mu \mathrm{Jy}$ (Lazarus et al. 2016).

The orbit of this DNS has a mild eccentricity of 0.0895 , and short orbital period of $4.95 \mathrm{hr}$. This places it among the most relativistic known DNS systems. Additionally, it is the most massive known DNS - our measurement of the advance of periastron $(\dot{\omega})$ is now found to be $5.6468 \pm 0.0013$ degrees per year corresponds to a total mass of $2.8862 \pm 0.0010 M_{\odot}$.

\section{Observations and results}

We have been observing PSR J1913+1102 with the Arecibo radio telescope since its 2012 discovery (Lazarus et al. 2016), in order to constrain its properties, with particular interest in its relativistic (i.e. post-Keplerian) orbital parameters. These data were acquired with the PUPPI pulsar backend system in coherent dedispersion mode. Observations were initially taken primarily at L-band, but profiles derived from these data displayed significant smearing due to interstellar scattering. We have therefore recently switched to using the higher frequency S-band receiver to counteract these large effects. 


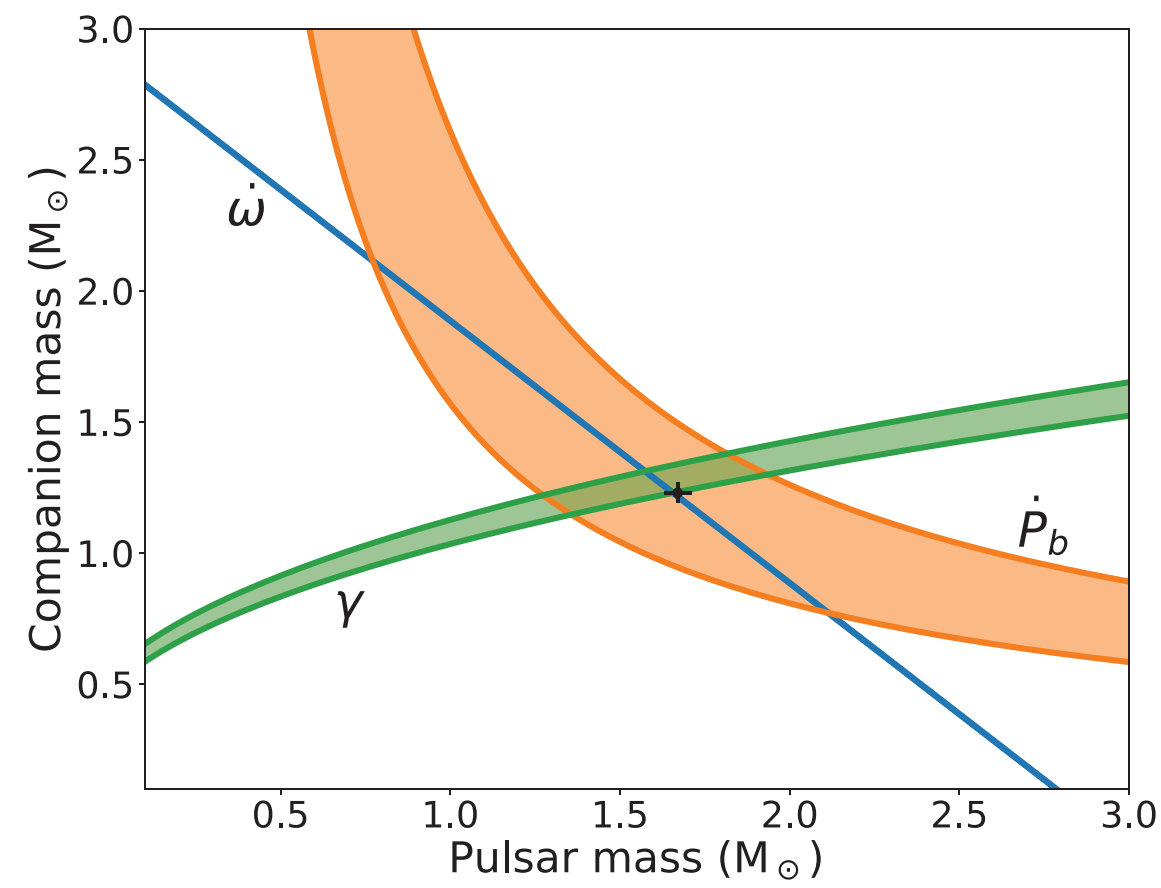

Figure 1. General relativistic-derived mass constraints for the pulsar and companion NS in the PSR J1913+1102 system. Each set of lines corresponds to the $1 \sigma$ uncertainties of the post-Keplerian parameters derived from timing analysis. These are: the rate of advance of periastron $\dot{\omega}$; the Einstein delay $\gamma$; and most recently found, the orbital decay rate $\dot{P}_{b}$. The masses of the pulsar and its companion as determined in the context of general relativity are shown as a black point, with corresponding uncertainties.

Indeed, the comparatively short time-spanm thus far of S-band observations has already provided similar timing precision to those in L-band with coherent dedispersion, but with far less systematic scattering effects.

The most recent year of observations have provided significant improvements to the measured orbital properties of PSR J1913+1102, particularly in its post-Keplerian parameters (see Figure 1). Specifically, in addition to the aforementioned improved $\dot{\omega}$ measurement, we have determined the Einstein delay $(\gamma)$, which is due to a combination of gravitational redshift and special relativistic time dilation. We measure this parameter to be $\gamma=0.472 \pm 0.020 \mathrm{~ms}$; combined with $\dot{\omega}$, this has allowed us to determine the masses of the pulsar and its NS companion to be $m_{p}=1.614 \pm 0.041 M_{\odot}$ and $m_{c}=1.273 \pm 0.041 M_{\odot}$, respectively.

These precise mass measurements confirm the asymmetric nature of the system, as originally speculated by Lazarus et al. (2016). This also leads to a prediction of the orbital decay, due to the emission of gravitational waves, of $\dot{P}_{b, \mathrm{GR}}=-0.460 \times 10^{-12} \mathrm{~s} \mathrm{~s}^{-1}$. We have now also detected the orbital decay in this system to be $\dot{P}_{b}=(-0.45 \pm 0.09) \times$ $10^{-12} \mathrm{~s} \mathrm{~s}^{-1}$, which is $1 \sigma$-consistent with the GR prediction. We expect that the precision of this measurement to now improve rapidly with further observations, since it scales as $T^{5 / 2}$, where $T$ is the time-span of observations. 


\section{Current and future implications}

Now that the large mass asymmetry of the system has been confirmed, PSR J1913+1102 holds great promise for several scientific endeavours, some of which have been discussed in the article reporting its discovery (Lazarus et al. 2016), and are outlined in what follows.

The stark mass asymmetry measured in the PSR J1913+1102 system is unique among the compact DNSs. This will allow us to set very stringent limits on the contributions to the measured decay rate from dipolar gravitational radiation, predicted by several alternative theories of gravity - but not by GR (e.g., Esposito-Farese 2005). PSR J1913+1102 may provide an even more precise such test than has been obtained from several NSwhite dwarf (WD) binaries, such as PSR J1141-6545 (Bhat, Bailes, Verbiest 2008), PSR J1738+0333 (Freire et al. 2012), and PSR J0348+0432 (Antoniadis et al. 2013). This is because in most pulsar-WD systems, the significance to which the WD mass is measured is generally limited by the precision from optical spectroscopy, and is therefore model-dependent. This is not the case for PSR J1913+1102 observations, which already yield considerably more constraining mass measurements than these NS-WD systems, and will continue to improve.

New insights into the formation and evolution of DNS systems can be gained from continued observations of PSR J1913+1102, and could further expand the range of possible NS birth masses. The relatively low companion mass and low eccentricity in the PSR J1913+1102 system hints at an electron-capture or symmetric supernova for the progenitor of the second-formed companion to PSR J1913+1102, as is believed to be the case for, e.g., PSRs J0737-3039A/B and J1756-2251 (Ferdman et al. 2013, 2014). As with those studies, we aim to observe PSR J1913+1102 over a sufficiently long time baseline in order to perform detailed profile shape evolution analysis. This will allow us to infer the system geometry, giving us further evidence regarding its evolutionary past, and the various channels through which DNS systems may form and populate our Galaxy.

In addition, a more precise proper motion measurement will help to characterise the natal kick imparted on this system by the second supernova. If this kick was small, then the system should have a small velocity relative to the Local Standard of Rest, and would lend support to the symmetric supernova scenario discussed above. Our current proper motion measurement is $\sim 10.6 \pm 1.8 \mathrm{mas} \mathrm{yr}^{-1}$, in the direction of the Galactic plane. This appears to be slightly larger than what has been measured for the double pulsar (Kramer et al. 2006; Deller et al. 2009), but still suffers from a lack of data. This measurement will also determine the extent to which apparent accelerations caused by proper motion effects balance those of Galactic acceleration when determining kinematic bias of the measured orbital decay rate. For a review of DNS formation theory, including preliminary constraints on the evolutionary history of PSR J1913+1102, see Tauris et al. (2017).

The PSR J1913+1102 system will increase the parameter space of expected coalescing $N S s$ to be observed by ground-based gravitational-wave observatories such as LIGO/VIRGO. Specifically, GW signals from events where there is tidal disruption of one of the NSs due to high mass asymmetry will yield unique information about the equation of state of dense matter, and will finally allow an estimate of the rate at which mergers of asymmetric DNS stars occur. The electromagnetic counterparts of such events are particularly important for understanding the Galactic heavy-element abundance (see, e.g., Tanvir et al. 2013; Just et al. 2015). 
Several similarities are seen in the properties of PSR J1913+1102 to those of the double pulsar system, PSR J0737-3039A/B - the only known pulsar binary in which both component NSs were discovered as emitting pulsars (Lyne et al. 2004). Specifically, its relatively low eccentricity, low companion mass, and short orbital period motivate the search for a companion pulsar. We have used the PUPPI backend at Arecibo to search for the companion, but no pulsed signal has yet been found. However, continued searches are well founded: the PSR J1913+1102 companion may be significantly more luminous at specific orbital phases, as was the case in the double pulsar system (Lyne et al. 2004; Kramer et al. 2006). It is also entirely plausible that the PSR J1913+1102 companion, if it is an emitting pulsar, may precess back into our line of sight at any moment. The discovery of a companion pulsar would provide additional GR tests and further insight into the formation and evolution of close DNS binaries.

\section{Conclusion}

Through longe-term observations of PSR J1913+1102, we have determined three postKeplerian parameters from high-precision timing analysis: advance of periastron, Einstein delay, and orbital decay rate. This has allowed us to determine the individual component masses in this DNS binary, for which we find a large asymmetry. The properties of this compact, relativistic binary appear to be very similar to other DNS systems in which we believe the second-born NSs were formed in a symmetric supernova event. In addition to binary evolution studies, we are encouraged that continued observations and analysis of PSR J1913+1102 will become a useful tool for setting limits on predictions made by alternative theories of gravity, and the study of DNS coalescence, of particular use to terrestrial gravitational-wave observatories.

\section{References}

Antoniadis, J., et al. 2013, Science, 340, 448

Bhat, R., Bailes, M., \& Verbiest, J. 2008, Phys. Rev. D, 77, 124017

\&Cordes, J. M. and Lazio, T. J. W. 2003, arXiv:astro-ph/0301598

Deller, A. T., Bailes, M., \& Tingay, S. J. 2009, Science, 323, 1327

Esposito-Farese, G. 2005, in M. Novello, S. Perez Bergliaffa, and R. Ruffini, editors, The Tenth Marcel Grossmann Meeting, page 647. World Scientific Publishing, 2005.

Ferdman, R. D., et al. 2013, ApJ, 767, 85

Ferdman, R. D., et al. 2014, MNRAS, 443, 2183

Freire, P. C. C., et al. 2012, MNRAS, 423, 3328

Just, O., et al. 2015, MNRAS, 448, 541

Kramer, M., et al. 2006, Science, 314, 97

Lazarus, P., et al. 2016, ApJ, 831, 150

Lyne, A. G., et al. 2004, Science, 303, 1153

Tanvir, N. R., et al. 2013, Nature, 500, 547

Tauris, T. M., et al. 2017, ApJ, 846, 170 